\title{
LUDUS - Desenvolvimento de um Jogo para Auxiliar no Aprendizado de Matemática para Alunos com Autismo
}

\author{
Diego de Abreu Porcellis \\ IFSul - Instituto Federal Sul-Rio- \\ Grandense \\ Bagé - RS - Brasil \\ diegoporcellis@ifsul.edu.br
}

\author{
Marcelo Siedler \\ IFSul - Instituto Federal Sul- \\ Rio-Grandense \\ Bagé - RS - Brasil \\ siedler@gmail.com
}

\author{
Marizele Garcia \\ IFSul - Instituto Federal Sul- \\ Rio-Grandense \\ Bagé - RS - Brasil \\ marizelegarcia@gmail.com
}

\begin{abstract}
The present work was created from the need to develop educational tools that can be used in the Multimedia Resource Rooms of the Bagé municipal education network, especially with children with autism. The software requirements were defined from meetings with the team of teachers and professionals who work in the Specialized Educational Service of General Emilio Luiz Mallet school, chosen as pilot of this project because it is one of the first schools to be contemplated with the classroom. resources. Among the listed requirements was prioritized to attend students aged 8-12 years and activities related to the four fundamental operations of mathematics: addition, subtraction, multiplication and division. As the purpose of the app is to support the teaching of students in the early grades of elementary school with autism. Thus, after the definition of the contents and the type of application, began to study the autistic universe and its particularities so that it was possible to design and develop an attractive game for this student.
\end{abstract}

\section{RESUMO}

O presente trabalho foi criado a partir da necessidade de desenvolvimento de ferramentas educacionais que possam ser usadas nas Salas de Recursos Multimídias da rede municipal de educação da cidade de Bagé, em especial, com crianças com autismo. Os requisitos do software foram definidos a partir de reuniões com a equipe de professores e profissionais que atuam no Atendimento Educacional Especializado da escola General Emilio Luiz Mallet, escolhida como piloto deste projeto por ser uma das primeira escolas a ser contemplada com a sala de recursos. Dentre os requisitos elencados foi priorizado atender a alunos com faixa etária de 8 a 12 anos de idade e atividades relacionadas às quatro operações fundamentais da matemática: adição, subtração, multiplicação e divisão. Como o objetivo do aplicativo é apoiar o ensino de alunos das séries iniciais do ensino fundamental com autismo. Desta forma, após a definição dos conteúdos e o tipo de aplicativo, passou-se a estudar o universo autista e suas particularidades para que fosse possível projetar e desenvolver um jogo atrativo para esse alunado.

\section{KEYWORDS}

Game, Autistic, Teaching, Mathematics

\section{Introdução}

A relação das pessoas com deficiência, vem se modificando de acordo, com a cultura, as crenças, fatores econômicos, políticos e sociais, para tal, vários documentos jurídicos foram criados, entre eles, a Lei Brasileira de Inclusão (LBI), como forma de assegurar e promover, em condições de igualdade, o exercício dos direitos e das liberdades fundamentais por pessoas com deficiência, visando sua inclusão social e cidadania. Frente a essa realidade, a inclusão de alunos com deficiência no ensino regular vem aumentando consideravelmente, tornando os recursos de Tecnologias Assistivas(TA) uma fonte de estímulo dessa parcela da sociedade, contribuindo para a ampliação do conhecimento, além de facilitar e enriquecer o processo de ensino e aprendizagem[1]. Uma parcela da sociedade que necessita das tecnologias assistivas são os alunos que possuem o Transtorno do Espectro Autista (autismo).

O autismo é uma condição neurobiológica que se manifesta antes dos três anos de idade e perdura por toda vida, afetando a área comunicativa, social e comportamental do indivíduo[2]. Muitas crianças com autismo apresentam grandes dificuldades de comunicação, onde o estilo responsivo de interação tem como princípio responder aos sinais e comunicações da criança para atender aos seus interesses e necessidades. Quanto mais motivada a criança estiver para interagir espontaneamente, maior será o seu envolvimento, participação e aprendizado.

Na cidade de Bagé, local onde este trabalho foi desenvolvido, tem se na rede municipal de ensino 630 alunos com necessidade de atendimento especializado e 36 destes apresentam transtornos do espectro autista segundo os dados apresentados ao censo escolar de 2014. 
Atualmente, o jogo é reconhecido como um aliado para o desenvolvimento das crianças, uma atividade importantíssima para desenvolver a capacidade de aprendizagem [3], um meio de expressão e maturação no plano físico, cognitivo, psicológico e social. Este elemento lúdico promove a flexibilização e expansão do pensamento, a curiosidade pelo novo, a criatividade e a expressividade, em um ambiente em que a criança se sente segura e confortável para explorar novas possibilidades, criar conexões, desenvolver novas habilidades. E o aprender brincando. Para Freitas [4] o conceito de jogo é um fazer ou uma participação do sujeito no meio, que lhe permite assimilar e incorporar a realidade. Segundo o autor, os jogos constroem uma grande rede de dispositivos que permitem à criança a assimilação de toda a realidade, incorporando-a para revivê-la, dominá-la e compensá-la.

Neste contexto, foi criado o +Ludus: MAth, um aplicativo voltado ao ensino de matemática para alunos do ensino fundamental com transtornos do espectro autista. O Aplicativo é um jogo digital que visa apresentar de forma lúdica exercícios que estimulem o aprendizado das quatro operações fundamentais da matemática. O aplicativo foi desenvolvido para ser multiplataforma, possibilitando, assim, sua utilização em computadores desktops, tablets e smartphones.

\section{Metodologia e Desenvolvimento}

A educação inclusiva no município de Bagé, dispõe de Salas de Recursos Multifuncionais (SRM) em todas as escolas de ensino fundamental (34), já nas escolas de educação infantil são 04 sala e nas demais (17) estão sendo implementadas. Neste espaço se propicia o Atendimento Educacional Especializado (AEE), considerado como um serviço de natureza didático-pedagógica que tem a função de identificar, elaborar e organizar recursos pedagógicos e de acessibilidade que eliminem as barreiras para a plena participação dos alunos, considerando as suas necessidades específicas. Contudo, o AEE complementa e/ou suplementa a formação do aluno com vistas à autonomia e independência na escola e fora dela.

Os sujeitos que se beneficiam do AEE, são alunos com deficiências física, intelectual e sensorial, os quais, em interação com diversas barreiras, podem obstruir sua participação plena e efetiva na sociedade em igualdade de condições com as demais pessoas, bem como, alunos com transtornos do espectro autista (autismo, síndrome de Asperger, síndrome de Rett) alunos com altas habilidades/superdotação, que apresentam um potencial elevado e grande envolvimento com as áreas do conhecimento humano, isoladas ou combinadas: intelectual, acadêmica, liderança, psicomotora, artes e criatividade.

O presente trabalho foi criado a partir da necessidade de desenvolvimento de ferramentas educacionais que possam ser usadas nas SRM da rede municipal de educação, em especial, com crianças com autismo. Os requisitos do software foram definidos partir de reuniões com a equipe de professores e profissionais que atuam no AEE da escola General Emilio Luiz Mallet, escolhida como piloto deste projeto por ser uma das primeira escolas a ser contemplada com a sala de recursos. Os principais requisitos elencados nesta etapa foram:

- Atender a alunos com faixas etárias de 8 a 12 anos de idade.

- Atividades relacionadas às quatro operações fundamentais da matemática: adição, subtração, multiplicação e divisão.

- Aplicativo multiplaforma, permitindo sua instalação em equipamentos dos laboratórios de informática e nos dispositivos móveis dos professores e alunos.

A utilização do aplicativo em dispositivos móveis utiliza a perspectiva BYOD (Bring Your Own Device) onde cada aluno pode ajustar e usar seus dispositivos móveis personalizados no seu próprio tempo, conforto e comodidade [5], vindo ao encontro das necessidades apontadas pelos professores de conseguir inserir os celulares dos alunos em uma atividade pedagógica, estimulando o uso dos dispositivos como uma ferramenta de estudo. Além disso, a disponibilidade de dispositivos móveis e a facilidade na aquisição por grande parte dos grupos socioeconômicos fornecem razões convincentes para a adoção do BYOD nas escolas [6][7]. Outro ponto a ser ressaltado é que as BYOD fornecem a possibilidade de continuidade nos contextos de aprendizagem escolar e doméstico [8].

Como o objetivo do aplicativo é apoiar o ensino de alunos das séries iniciais do ensino fundamental, foi pensado na criação de um jogo que instigasse os alunos a interagir com aplicativo de forma divertida. Desta forma, após a definição dos conteúdos e o tipo de aplicativo, passou-se a estudar o universo autista e suas particularidades para que fosse possível projetar e desenvolver um jogo atrativo para os alunos.

Nesse período foram definidas algumas características que o jogo deveria ter, tais como, o jogo ser bastante colorido, ter como tema assuntos de interesse dos alunos na faixa etária definida, com personagens atrativos que estimulem o aluno a jogar, não passar mensagem negativa quando o aluno comete algum erro, evitando que o aluno se frustre e desista da atividade. Neste caso, se a resposta não estiver correta o usuário apenas deixa de ganhar a recompensa maior e recebe um aceno do personagem, tendo a possibilidade de tentar novamente.

\subsection{Jogo}

O jogo inicia com o aluno podendo escolher o personagem com o qual deseja jogar. São disponibilizados oito personagens, quatro meninas e quatro meninos, conforme apresentado na Figura 1. A partir desse ponto o usuário tem três opções, jogar, ir para a loja 
entrar nas configurações do jogo. Na loja são apresentados os cenários que o usuário pode adquirir com as moedas que ele acumula acertando as questões propostas pelo jogo. O cenário padrão é o da fazenda (Figura 2) podendo desbloquear outros quatro: Egito, Fadas, Mundo Marinho e Orcs.

Na seção de configurações é possível definir a orientação da operação matemática (vertical ou horizontal), essa funcionalidade foi incluída no jogo por sugestão de um pai de aluno pois, na escola as crianças estavam aprendendo as operações matemáticas na vertical e o jogo, inicialmente, só realizava operações na horizontal e acabava confundindo o seu filho, além disso é possível também, escolher o nível das expressões que serão apresentadas. $\mathrm{O}$ jogo oferece três níveis, sendo:

- Nível 1: expressões com até dois dígitos, o jogador deve acertar o resultado da operação.

- Nível 2: expressões com até dois dígitos, porém a resposta pode estar em qualquer lugar da operação, exemplo: ? + $3=5,4+?=9$

- Nível 3: expressões com três dígitos, o jogador deve acertar o resultado da operação.

O jogo consiste em resolver expressões matemáticas usando as quatro operações fundamentais e, para cada nível de dificuldade, são apresentadas cinco fases que são desbloqueadas à medida que o usuário vai avançando, tendo a seguinte sequência: adição, subtração, multiplicação, divisão e a fase final com expressões aleatórias, podendo ser de qualquer uma quatro operações fundamentais.

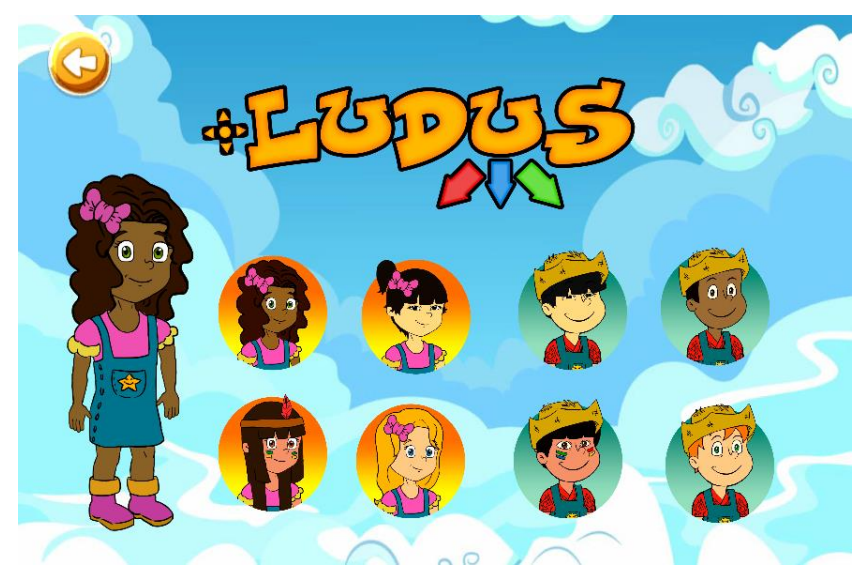

Figura 1: Tela de seleção de personagens

Para criação do jogo utilizou-se o ambiente Unity, e todo projeto foi desenvolvido por alunos do curso técnico integrado em informática, sendo o processo de criação da arte e programação do jogo um desafio para professores e alunos, pois este foi o primeiro jogo desenvolvido na instituição, sendo necessário estudar as particularidades do desenvolvimento de jogos digitais, que envolvem desde criação dos artefatos visuais do jogo, como menu, cenário e personagens, ate a integração com a ferramenta, com a criação das animações, sprites, disposição e relação dos elementos nas cenas e integração com os scripts criados.

O jogo apresenta uma versão estável para os sistemas operacionais Android, Windows, IOS e Ubuntu, permitindo a execução de todas funcionalidades propostas no projeto. Os testes de validação foram inicialmente realizados pela equipe do projeto, com o auxílio de professores do curso técnico em Informática e, posteriormente, tendo a versão para Windows instalada e utilizada por alunos da escola parceira (Figura 3).

Em parceria com a secretaria municipal de educação pretende-se levar o trabalho a todas as escolas do município, realizando a instalação nos laboratórios e equipamentos móveis disponíveis.

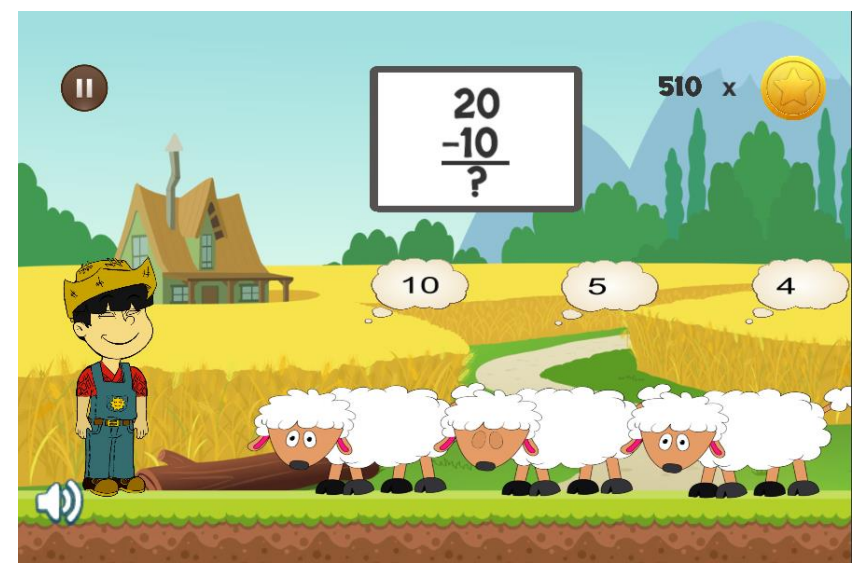

Figura 2: Operação de subtração no cenário Fazenda

\section{Considerações Finais}

O presente projeto apresentou o desenvolvimento de um software que pretende auxiliar na prática das operações fundamentais da matemáticas por alunos atendidos na sala de recursos da rede municipal de educação. O público alvo inicial do jogo foram alunos autistas. Porém, conforme os testes iniciais realizados na escola parceira, alunos com déficit de atenção e mesmo alunos regulares utilizaram o aplicativo, que deve ser colocado nas práticas realizadas pelos professores de matemática das séries iniciais.

Os testes iniciais do projeto foram realizados na instituição parceira do projeto, através da instalação no laboratório da escola e apresentação aos alunos das séries iniciais. Nesta etapa foi validado se o programa rodaria adequadamente no laboratório da escola e as primeiras impressões da utilização por parte alunos.

O objetivo é fazer uma análise qualitativa do aplicativo na escola parceira, porém, o laboratório da escola apresentou problemas 
estruturais e ainda não foi possível realizar a continuidade dos testes. A equipe do projeto está em tratativas com a secretaria municipal de educação para viabilizar os testes na escola e, posteriormente, disponibilizar o jogo gratuitamente para a comunidade através das lojas de aplicativos virtuais.

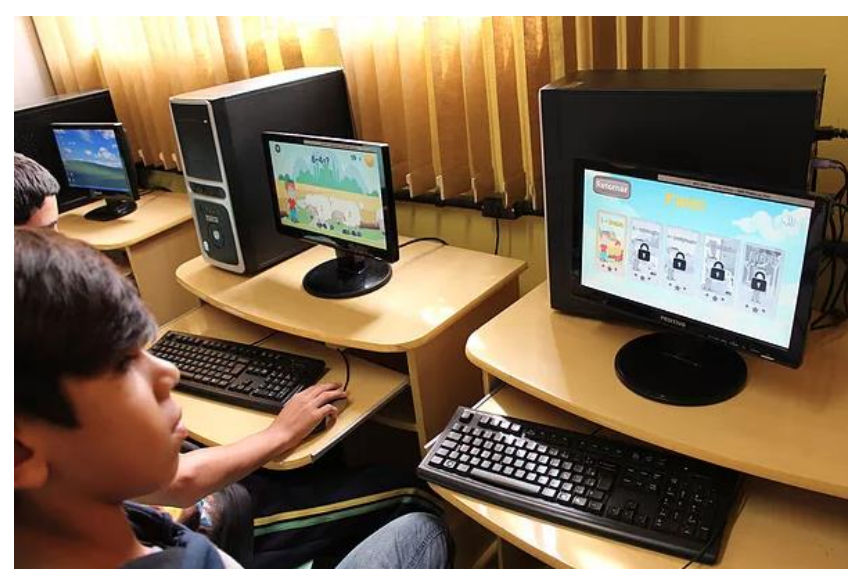

Figura 3: Alunos do ensino fundamental utilizando o aplicativo no laboratório de informática.

Como trabalhos futuros pretende-se realizar treinamento com os professores da rede pública, ensinando o funcionamento e instalação do jogo, além de uma análise da sua utilização em sala de aula, sob a ótica do aluno com deficiência e dos professores do AEE.

\section{REFERÊNCIAS}

[1] Martins, S. G., Doumany, S. J., Uchoa M. E., A rrelias D. J. E Leite F. W. E. 2016 Literal Azul: Protótipo de software de apoio a alfabetização de autistas", Anaisdos W orkshops do V Congresso Brasileiro de Informática na Educação. p. 205-210.

[2] Felicio, V. C. (2007) "O Autismo e o Professor: Um saber que pode Ajudar", Universidade Estadual Paulista Júlio de Mesquisa FilhoFaculdade de CiênciasCampus de Bauru. Bauru/SP.

[3] Tezani ${ }^{1}$, Thaís Cristina R. (2006) 0 jogo e os processos de aprendizagem e desenvolvimento: aspectos cognitivos e afetivos. Educação em revista, 7.1-2:1-16.

[4] Freitas, M.T.A. de. Vygotsky e Bakhtin: Psicologia e Educação: um intertexto. São Paulo: Editora Á tica, 2000.

[5] Pereira, A. B., Piconez, S., Zimmer, J., \& da Silva, F. S. C. (2016, November). Jogos Digitais no desenvolvimento de conceitos matemáticos sob perspectiva BYOD e abordagem m-Learning na escola pública. In Anais do Workshop de Informática na Escola (Vol. 22, No. 1, p. 71)

[6] Johnson, D. (2012). A bordo com BYOD. Educ. Leadersh. 70, 84-85.

[7] Stavert, B. (2013). Traga seu próprio dispositivo (BYOD) nas escolas: revisão de literatura de 2013 . Sydney, NSW: Departamento de Educação e Comunidades de New South Wales.

[8] Lai, KW, Khaddaget, F. e Knezek, G. (2013). Misturando experiências de tecnologia dos alunos na aprendizagem formal $\mathrm{e}$ informal. J. Comput. Assist. Aprender. 29, 415-425. doi: 10.1111 / jcal.12030 